\title{
miR-142-3p regulates the formation and differentiation of hematopoietic stem cells in vertebrates
}

\author{
Xinyan $\mathrm{Lu}^{1, *}$, Xiajuan $\mathrm{Li}^{1, *}$, Qiuping $\mathrm{He}^{1}, \mathrm{Jiao} \mathrm{Gao}^{2}$, $\mathrm{Ya} \mathrm{Gao}^{1}$, Bing $\mathrm{Liu}^{2}$, Feng $\mathrm{Liu}^{1}$ \\ ${ }^{I}$ State Key Laboratory of Biomembrane and Membrane Biotechnology, Institute of Zoology, Chinese Academy of Sciences, Beijing \\ 100101, China; ${ }^{2} 307-I v y$ Translational Medicine Center, Laboratory of Oncology, Affiliated Hospital of Academy of Military Medi- \\ cal Sciences, Beijing 100071, China
}

Previous studies on developmental hematopoiesis have mainly focused on signaling and transcription factors, while the appreciation of epigenetic regulation including that of microRNAs is recent. Here, we show that in zebrafish and mouse, $m i R-142-3 p$ is specifically expressed in hematopoietic stem cells (HSCs). Knockdown of miR-142a$3 p$ in zebrafish led to a reduced population of HSCs in the aorta-gonad-mesonephros (AGM) region as well as T-cell defects in the thymus. Mechanistically, miR-142a-3p regulates HSC formation and differentiation through the repression of interferon regulatory factor 7 (irf 7 )-mediated inflammation signaling. Finally, we show that miR-142-3p is also involved in the development of HSCs in mouse AGM, suggesting that it has a highly conserved role in vertebrates. Together, these findings unveil the pivotal roles that $m i R-142 a-3 p$ plays in the formation and differentiation of HSCs by repressing $i r f 7$ signaling.

Keywords: hematopoietic stem cell; miR-142a-3p; irf7; zebrafish; mouse

Cell Research (2013) 23:1356-1368. doi:10.1038/cr.2013.145; published online 29 October 2013

\section{Introduction}

Genetic control over the development of hematopoietic stem cells (HSCs) is highly conserved in vertebrates, thus allowing the use of model organisms, such as zebrafish and mouse, to elucidate the regulatory mechanisms underlying HSC programming [1, 2]. In zebrafish, HSCs emerge from the ventral wall of the dorsal aorta via an endothelial-to-hematopoietic transition (EHT) [3, 4], migrate to the caudal hematopoietic tissue (CHT) and finally home to the thymus and kidney to produce $\mathrm{T}$ cells and other blood cell lineages [2]. The self-renewal and differentiation of HSCs ensure a lifelong supply of hematopoietic cells to meet the demand and to renew blood cells in organs, which must be tightly controlled at the cellular and molecular levels. However, the underlying molecular mechanisms of HSC specification and differ-

\footnotetext{
*These two authors contributed equally to this work. Correspondence: Feng Liu

Tel: +86 (10) 64807307; Fax: +86 (10) 64807313

E-mail: liuf@ioz.ac.cn

Received 16 July 2013; revised 19 August 2013; accepted 17 September 2013; published online 29 October 2013
}

entiation during vertebrate embryogenesis are still poorly understood.

microRNAs (miRNAs) are a group of non-coding RNAs ( $22 \mathrm{nt})$ that can silence gene expression by binding to the $3^{\prime}$ untranslated region (UTR) or to the coding region of target mRNAs to promote mRNA destabilization or inhibit protein translation $[5,6]$. Importantly, many miRNAs have been shown to be expressed in hematopoietic lineages and to act as pivotal regulators of transcriptional programs for normal hematopoiesis, including HSC self-renewal, differentiation and functioning $[7,8]$. For example, miR-126 is highly expressed in human and mouse HSCs, and the attenuation of $m i R$ 126 activity by lentivirus-mediated knockdown leads to the inexhaustible expansion of HSCs in vivo in mouse and human [9]. Zebrafish $m i R-126$ was reported to be necessary for the precise regulation of the erythroid/ megakaryocytic lineage choice during definitive hematopoiesis in vivo [10]. In addition, $m i R-144$ and $m i R-451$, as direct targets of the critical hematopoietic master regulator GATA1, are required for erythropoiesis in zebrafish and mouse [11-13]. $m i R-142-3 p$ is an evolutionally conserved miRNA of vertebrates, which is expressed in many different hematopoietic cells [14-21]. Chen et al. 
[15] first reported that miR-142 is expressed in embryonic and adult hematopoietic tissues, including the fetal liver, bone marrow, spleen and thymus, in mice, indicating its role in both embryonic and adult hematopoiesis. Later studies using mouse and human cell lines have indicated that $m i R-142-3 p$ is required for the development and function of terminally differentiated hematopoietic lineages, such as myeloid and dendritic cells. miR-142$3 p$ can directly regulate the expression of tab2 in the myeloid lineage [22] and that of $I L-6$ in dendritic cells(DCs) [23]. Very recently, zebrafish miR-142-3p (ZFIN ID: ZDB-GENE-090929-151, miR-142a-3p herein) has been shown to be required for the maturation of primitive erythrocytes, cardiogenesis and vessel integrity [24, 25]. However, the mechanisms underlying miRNA regulation of HSC development in vivo during vertebrate embryogenesis remain largely unclear.

Here, we show that in zebrafish, $m i R-142 a-3 p$ controls HSC specification and differentiation by suppressing the expression of interferon regulatory factor 7 (irf7), revealing the pivotal roles of $m i R-142 a-3 p$ in HSC develop- ment. Moreover, $m i R-142-3 p$ is also involved in the development of HSCs in mouse aorta-gonad-mesonephros (AGM) both in vitro and in vivo, suggesting that it has a well-conserved role in vertebrates.

\section{Results}

miR-142-3p is evolutionarily conserved and expressed in HSCs during zebrafish embryogenesis

$m i R-142-3 p$ is highly conserved in vertebrates, from zebrafish to mammals (Supplementary information, Figure S1A). During zebrafish embryogenesis, miR-142a$3 p$ expression was detected by RT-PCR from the tailbud stage onward (Supplementary information, Figure $\mathrm{S} 1 \mathrm{~B})$. Whole mount in situ hybridization (WISH) showed that $m i R-142 a-3 p$ is expressed at low levels in the AGM region at 24 hour post fertilization (hpf), where the first HSCs will emerge 6-10 h later [3] (Figure 1A). Using the transgenic runx 1:EGFP (Zhang P and Liu F, unpublished data) and $c m y b$ :EGFP [26] lines, $m i R-142 a-3 p$ was found to be highly expressed in GFP-positive cells, which rep-
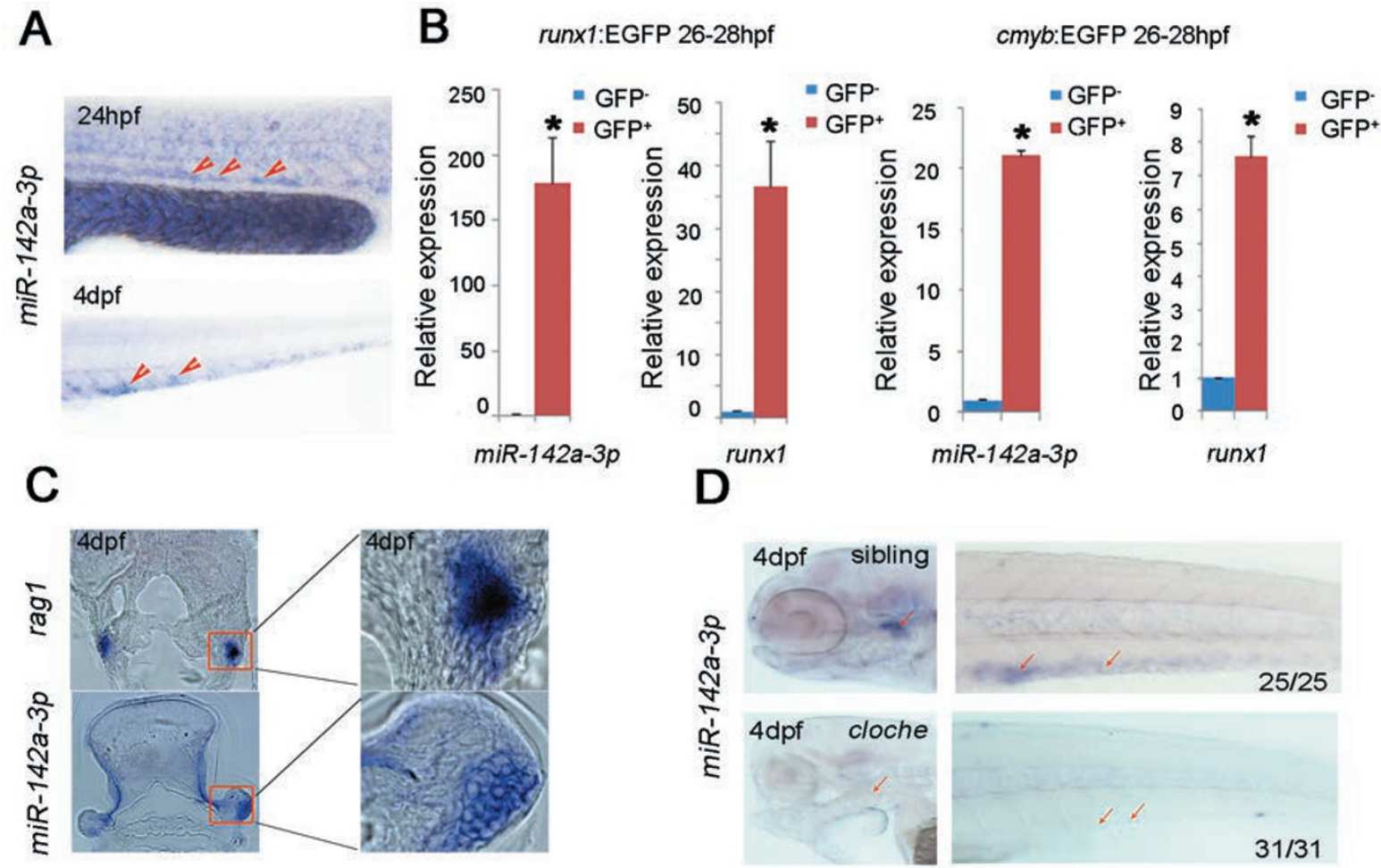

Figure 1 miR-142a-3p is specifically expressed in HSCs and T cells in zebrafish. (A) WISH images showing miR-142a-3p expression in the AGM (red arrowheads) at $24 \mathrm{hpf}$ and in the CHT (red arrowheads) at $4 \mathrm{dpf}$. (B) qPCR results showing the expression of miR-142a-3p in sorted runx1:EGFP- or cmyb:EGFP-positive HSCs vs EGFP-negative cells. runx1 was used as a positive control. (C) Frozen sections showing the expression of rag1 and miR-142a-3p in the thymus at 4 dpf. The red square marks the thymus region. (D) WISH images showing the absence of miR-142a-3p expression in the thymus and $\mathrm{CHT}$ of the cloche mutants compared with WT siblings at $4 \mathrm{dpf}$. 
resent the population of hematopoietic stem/progenitor cells, at 26-28 hpf (Figure 1B). Starting from $48 \mathrm{hpf}$ and onwards, $m i R-142 a-3 p$ was specifically expressed in the $\mathrm{CHT}$ and at 4 day post fertilization (dpf) in the thymus (Figure 1A and 1C). Immunostaining of cryosections of 4 dpf embryos further confirmed that $m i R-142 a-3 p$ was expressed in the thymus (Figure 1C). In contrast, the expression of $m i R-142 a-3 p$ was not detected in either the thymus or the CHT region of cloche mutants that lack hematopoietic cells (Figure 1D), indicating that $m i R-142 a$ $3 p$ is indeed expressed in hematopoietic cells. The specific hematopoietic expression of $m i R-142 a-3 p$ suggests that it might play a critical role in definitive hematopoiesis.

Loss of miR-142a-3p leads to defects in HSC development and T-cell differentiation

To interrogate the function of $m i R-142 a-3 p$, we analyzed the effects of blocking the formation of mature $m i R$ $142 a-3 p$ by using two different antisense morpholinos (MOs) targeting different sites of the miR-142a-3p stem loop, $3 p \mathrm{MO} 1$ and $3 p \mathrm{MO}$, and a mismatch morpholino of $m i R-142 a-3 p$, mis $3 p \mathrm{MO}$, was used as a control (Figure 2A and Supplementary information, Table S1). To exclude the possibility that developmental delay was caused by the morpholino injections, we examined the expression of the somite marker myod and found that its expression levels were not obviously different in the controls and morphants at $24 \mathrm{hpf}$ (Supplementary information, Figure S2A). Additionally, the expression of the neuron and pronephric duct marker pax2.1 was also not altered (Supplementary information, Figure S2A). These data suggest that at 24 hpf, the morphants grew relatively normally after the $m i R$ $142 a-3 p$ knockdown. qPCR showed that the endogenous expression of $m i R-142 a-3 p$ was reduced to various degrees (2-7 folds) by $3 p$ MO1 or $3 p$ MO2 injection (at 3.2 ng per embryo) (Figure 2A). WISH using a locked nucleic acid (LNA) antisense probe against mature miR-142a-3p revealed that the expression of $m i R-142 a-3 p$ in the thymus and the CHT was almost undetectable in the miR-142a$3 p$ morphants and that the suppression effect continued through $4 \mathrm{dpf}$ (Supplementary information, Figure S2B and $\mathrm{S} 2 \mathrm{C}$ ). Thus, the hematopoietic expression of $m i R$ $142 a-3 p$ was efficiently suppressed by both MOs (Figure $2 \mathrm{~A}$, Supplementary information, Figure S2B and S2C). Interestingly, the expression of $m i R-142 a-5 p$, which originates from the opposite strand of the miR-142a gene, was detectable only in the thymus but not in the AGM region, and its expression in the thymus was also reduced by both of the miR-142a-3p MOs (data not shown).

To test whether miR-142a-3p influences HSC development and differentiation, we examined the expression of multiple markers of HSCs and hematopoietic lineages, such as the HSC genes runxl and cmyb and the T-cell markers ragl and ikaros. The WISH and qPCR results showed that the miR-142a-3p morphants (generated by injection of $3 p \mathrm{MO} 1$ or $3 p \mathrm{MO} 2$ ) displayed decreased expression of runx 1 and $c m y b$ in the AGM and CHT at various stages (Figure 2B, $2 \mathrm{C}$ and Supplementary information, Figure S3A), whereas the miR-142a-5p knockdown did not have any effect on runxl expression in the AGM (data not shown), suggesting that $m i R-142 a-3 p$, but not $m i R-142 a-5 p$, is required for HSC development in zebrafish. Moreover, at $4 \mathrm{dpf}$, the expression of the $\mathrm{T}$ -cell markers ragl and ikaros was also decreased, whereas the expression of the thymic epithelial cell markers foxn 1 and ccl25a remained unchanged (Figure 2E, 2F and Supplementary information, Figure S3A-S3C), suggesting that the decrease in the T-cell population resulting from the loss of $m i R-142 a-3 p$ expression is not attributable to a defective thymic epithelial microenvironment. Furthermore, knockdown experiments performed using the $c m y b$ :EGFP transgenic line showed that the miR$142 a-3 p$ morphants had significantly reduced $c m y b^{+}$HSC populations in both the AGM and CHT regions (Figure 2D). In contrast, embryos injected with mismatch MOs displayed normal expression of the HSC markers runxl and $c m y b$, and the T-cell marker ragl (Figure 2B, 2C and Supplementary information, Figure S3A). To further confirm that the defects in HSC development in the morphants truly resulted from miR-142a-3p knockdown, we tested whether a $m i R-142 a-3 p$ duplex can rescue HSC gene expression in the miR-142a-3p morphants. Indeed, WISH showed that the decreased runxl expression in the morphants was rescued by the duplex (Figure 2G). Time-course analysis revealed the continuous decrease in $c m y b$ expression in the AGM and CHT of the morphants from 36 hpf to $5 \mathrm{dpf}$ (Supplementary information, Figure $\mathrm{S} 3 \mathrm{D})$, further supporting that this defect in HSC development in the miR-142a-3p morphants was not due to developmental delay. Together, these data demonstrate that $m i R-142 a-3 p$ is required for HSC development and T-cell differentiation.

\section{Hemogenic endothelium but not arterial programming is affected in miR-142a-3p morphants}

The decreased expression of the HSC master regulators runxl and $c m y b$ in the miR-142a-3p morphants indicates that HSC emergence and development were severely disrupted. The first HSCs emerge in the ventral wall of the dorsal aorta (i.e., the hemogenic endothelium) via an EHT process, and arterial specification is a prerequisite for the normal emergence of HSCs [1]. To test whether the arterial programming was affected, we examined the expression of the arterial marker $\operatorname{dlt} C$ and 

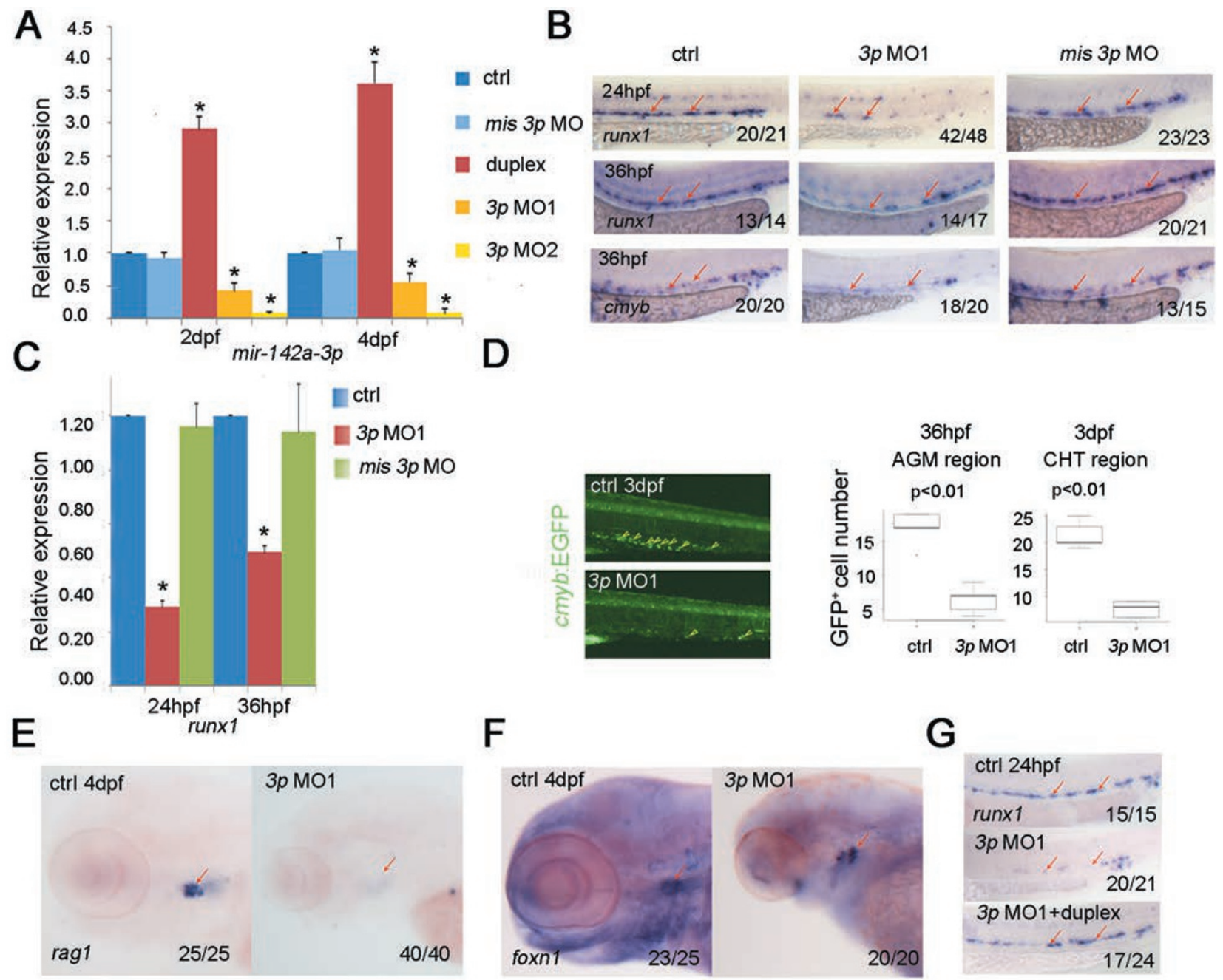

$G$

Figure 2 Loss of miR-142a-3p leads to defects in HSC development and T-cell differentiation in zebrafish. (A) Expression of $m i R-142 a-3 p$ in embryos injected with the miR-142a-3p mismatch MO, duplex, or two different miR-142a-3p MOs, at 2 and 4 $\mathrm{dpf}$ (mean $\pm \mathrm{SD}$, $t$-test, ${ }^{*} P<0.05, n=3$ ). Total RNA extracted from embryos at 2 and 4 dpf was subjected to qPCR, with $U 6$ serving as the internal control. (B) WISH images showing the decreased expression of the HSC master regulators runx1 and cmyb in miR-142a-3p morphants, but no changes in the embryos injected with mis $3 p$ MOs. (C) qPCR results showing the decreased runx 1 expression in miR-142a-3p morphants, but no changes in embryos injected with mis $3 p$ MOs at 24 and 36 hpf (mean $\pm \mathrm{SD}, t$-test, ${ }^{*} P<0.05, n=3$ ). (D) miR-142a-3p knockdown in cmyb:EGFP transgenic zebrafish embryos led to a decreased $c m y b^{+}$population. Left panel, representative fluorescence images showing the $c m y b^{+}$population in the $\mathrm{CHT}$ at 3 dpf. Right panels, quantitative analyses of the $c m y b^{+}$populations in the AGM (36 hpf) and CHT ( $3 \mathrm{dpf}$ ), showing a significant difference between the miR-142a-3p morphants and the controls (mean $\pm \mathrm{SD}, t$-test, $P<0.01, n=3$ ). (E) Decreased expression of the T-cell marker rag1 in miR-142a-3p morphants at $4 \mathrm{dpf}$. The red arrows indicate the thymus. (F) Unchanged expression of the thymic epithelial cell marker foxn1 in miR-142a-3p morphants at $4 \mathrm{dpf}$. The red arrows indicate the thymus. (G) Restoration of runx1 expression by miR-142a-3p duplex in miR-142a-3p morphants at $24 \mathrm{hpf}$. The red arrows indicate the HSCs in the AGM region.

the venous marker $f t 4$ using WISH. The results showed that the expression of $d l t C$ and $f t t 4$ was unchanged in the morphants (Figure 3A), suggesting that arterial/venous programming is not disrupted. Furthermore, a $3 p$ MO1 injection experiment using flila:EGFP transgenic zebrafish showed that the dorsal aorta is normal in the mor- phants, compared with that of the controls (Figure 3B).

The decrease of runxl expression at $24 \mathrm{hpf}$ in the AGM region of $m i R-142 a-3 p$ morphants (Figure $2 \mathrm{~B}$ and Supplementary information, Figure S3A) indicated that the specification of the hemogenic endothelium might be affected. To verify this hypothesis, we used the double- 
transgenic line $k d r l: m C h e r r y / c m y b$ :EGFP in which the double-positive cells (yellow cells) represent hemogenic endothelial cells [4]. Indeed, at $36 \mathrm{hpf}$, the number of $k d r l^{+}{ }^{c m y b} b^{+}$cells was reduced in the miR-142a-3p morphants, which persisted until $60 \mathrm{hpf}$, the latest stage that we monitored (Figure 3C). The reduction of the hemogenic endothelial cell population was unlikely due to dysregulated cell proliferation or cell-cycle arrest (data not shown). Therefore, considering the high expression of $m i R-142 a-3 p$ in the sorted $c m y b^{+}$or runx $1^{+}$cells at 26$28 \mathrm{hpf}$ in wild-type (WT) zebrafish (Figure 1B), and the decrease of runxl expression at $24 \mathrm{hpf}$ (Figure 2B and Supplementary information, Figure S3A) as well as the reduced number of $\mathrm{kdrl}^{+} \mathrm{cmyb} \mathrm{b}^{+}$cells at $36 \mathrm{hpf}$ in $\mathrm{miR}$ $142 a-3 p$ morphants (Figure 3C), we concluded that the $m i R-142-3 p$ knockdown affected hemogenic endothelial specification, which in turn attenuated the emergence of the earliest HSCs in the embryos.

Expression profiling identified downstream targets of miR-142a-3p

Direct comparison of the global gene expression patterns between control and miR-142a-3p-knockdown embryos might reveal further clues toward elucidating the biological effects of $m i R-142 a-3 p$ on HSC formation and differentiation. To identify the direct downstream targets of $m i R-142 a-3 p$, we performed a combination of microarray, RT-PCR and miRNA target prediction (Figure 4A$4 \mathrm{C}$ and Supplementary information, Figure S4). The total RNAs of the AGM region in controls and miR-142a-3p morphants at both $2 \mathrm{dpf}$ and $4 \mathrm{dpf}$ were extracted and reverse-transcribed. The Cy3- or Cy5-labeled cDNAs were hybridized to a zebrafish oligo microarray. Gene set enrichment analysis was applied to the gene expression data to identify $m i R-142 a-3 p$-modulated signaling and gene expression (Figure 4A). In total, 70 genes were upregulated at both $2 \mathrm{dpf}$ and $4 \mathrm{dpf}$ (Figure 4B). Based on combined target-prediction analyses using Pictar and TargetScan, four potential miR-142a-3p targets, irf7, krt18, foxpla and rxrab were further analyzed in detail using RT-PCR, qPCR and WISH (Supplementary information, Figure S4A-S4C). The results showed that among these four genes, the expression of irf7 was dramatically upregulated in miR-142a-3p morphants compared to controls (Supplementary information, Figure S4A and S4B), suggesting that $i r f 7$ may be a primary target of $m i R$ $142 a-3 p$ in HSCs and their differentiated lineages. Although irf7 is one of the components of interferon signaling, its role in HSC development has not been reported. Therefore, we chose irf7 for functional analysis.

irf7 is a direct target of miR-142a-3p

irf7 has an imperfect match with $m i R-142 a-3 p$, which

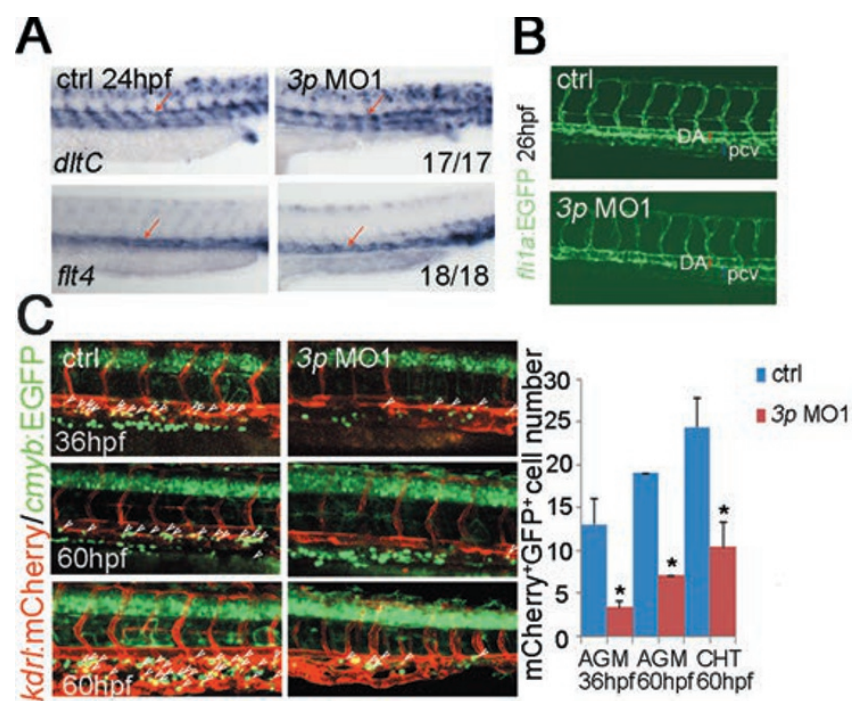

Figure 3 Loss of miR-142-3p did not affect artery/vein identity, but affected hemogenic endothelial specification. (A) WISH images showing the unchanged expression of arterial marker dltC and venous marker flt4 in miR-142a-3p morphants at $24 \mathrm{hpf}$. (B) Confocal microscopy showing the unaffected artery and vein development in $m i R-142 a-3 p$ morphants using fli1a:EGFP transgenic zebrafish. DA, dorsal aorta. (C) Decreased number of hemogenic endothelial cells in the AGM as well as decreased HSCs in the CHT of miR-142a-3p morphants produced using the $k d r l: m C h e r r y / c m y b$ :EGFP transgenic line. Right panel, quantitative analyses of the $k d r^{+} / \mathrm{cmyb}^{+}$populations showed a significant difference between the miR-142a-3p morphants and the controls (mean $\pm \mathrm{SD}, t$-test, ${ }^{*} P<0.05, n=3$ ).

potentially targets residues 312 to 339 of the $\operatorname{irf7} 3^{\prime}$ UTR (with a minimum free energy (MFE) of $-24.3 \mathrm{kcal} / \mathrm{mol}$; RNAHybrid Website: http://bibiserv.techfak.uni-bielefeld.de/rnahybrid/), but has no match with $m i R-142 a-5 p$ (Figure 4C and data not shown). qPCR results showed that $\operatorname{irf} 7$ expression level was markedly increased in the $m i R-142 a-3 p$ morphants at $2 \mathrm{dpf}$ and $4 \mathrm{dpf}$ (Supplementary information, Figure S4B). To determine whether $m i R-142 a-3 p$ regulates irf7 expression directly or indirectly, we fused the zebrafish irf7 3' UTR containing the putative $m i R-142 a-3 p$ recognition site or mutated sites (mu1 or mu2) with a luciferase reporter (Figure 4D), and transfected the reporter together with the miR-142a$3 p$ duplex into HEK293T cells. Luciferase activity was strongly repressed when $m i R-142 a-3 p$ was co-expressed with the WT pGL3-irf7-3' UTR but not when it was coexpressed with the mutant ones (Figure 4E). Western blotting showed that the expression level of $i r f 7$ was decreased in duplex-injected embryos (Figure 4F). Taken together, these results suggest that $m i R-142 a-3 p$ directly binds to the $\operatorname{irf} 73^{\prime}$ UTR region in a sequence-specific manner to repress irf7 expression. 
A

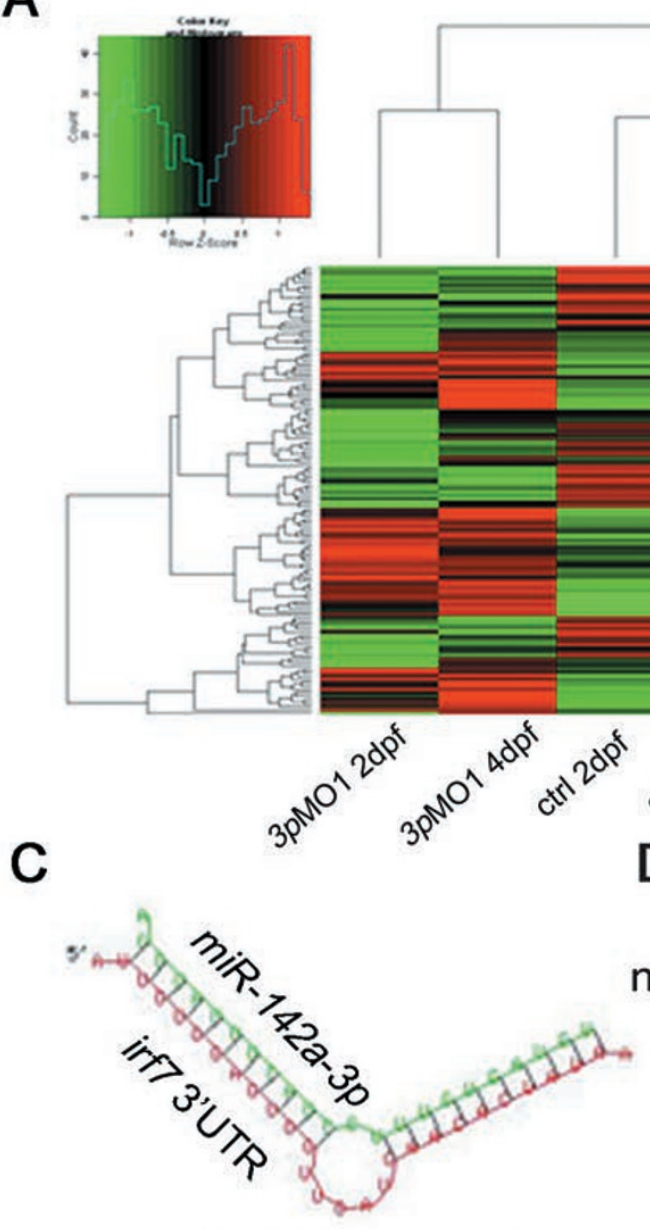

mfe:-24.3 kcal/mol
B

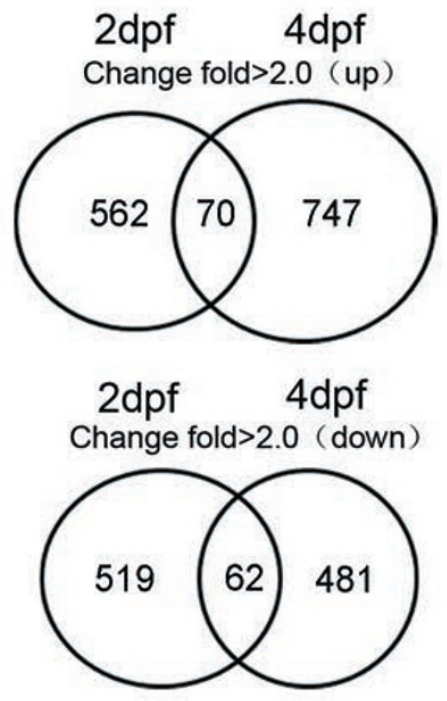

E

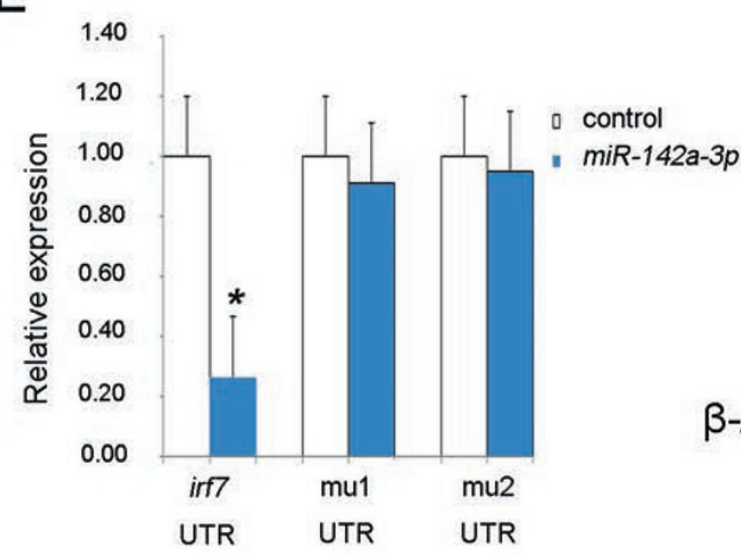

$\mathbf{F}$

\begin{tabular}{|l|l|l|l|}
\hline SV40 & luciferase & irf7 & polyA \\
\hline SV40 & luciferase & irf7 & polyA
\end{tabular}

wt ATTGTGGAGTGGTTGATGAACACTATA

mu1 ATTGTGG TTGATGAACACTATA

wt ATTGTGGAGTGGTTGATGAACACTATA mu2 ATTGTGGAGTGGTTGATG

Figure 4 irf7 is a direct target of miR-142a-3p. (A) Heat map analysis of gene expression in controls and miR-142a-3p morphants at $2 \mathrm{dpf}$ and $4 \mathrm{dpf}$. (B) Fold changes of upregulated or downregulated genes at $2 \mathrm{dpf}$ and $4 \mathrm{dpf}$ in miR-142a-3p morphants compared to controls. (C) An imperfect match of irf7 3' UTR with miR-142a-3p as determined by a calculation using RNAHybrid. (D) Scheme of the PGL3 constructs containing irf7 WT and mutated 3' UTR. (E) Luciferase reporter activity of the reporter containing WT or mutated irf7 3' UTR when co-transfected with the miR-142a-3p duplex into HEK293T cells (mean $\pm \mathrm{SD}, t$-test, ${ }^{*} P<0.05, n$ = 3). (F) Western blotting images showing that irf7 protein level was decreased in duplex-injected embryos at $4 \mathrm{dpf}$, compared to controls (left panel). Quantitative analysis of the western blotting results is shown in right panel. 
To determine whether irf7 is responsible for the miR$142 a-3 p$ morphant phenotypes, we employed a doubleknockdown approach. Importantly, double knockdown by co-injecting irf7 MO and $3 p$ MO1 partially rescued the expression of runx 1 at $24 \mathrm{hpf}$ and that of $c m y b$ at 36 hpf (Figure 5A). Furthermore, the double knockdown of $m i R-142 a-3 p$ and irf7 clearly rescued the expression of the T-cell marker ragl (Figure 5A), suggesting that irf7 is required for $m i R-142 a-3 p$-regulated lineage differentiation. Western blotting confirmed that co-injecting irf7 MO with $3 p$ MO1 restored the Runx 1 protein level at 24 hpf (Figure 5B), which provided further evidence for the rescue of defects in HSC development by knocking down $i r f 7$ in the miR-142a-3p morphants.

Decreased Gcsfr-Nitric Oxide (NO) inflammation signaling might lead to defects in HSC development in the miR142a-3p morphants

To elucidate the mechanism underlying the $m i R-142 a-3 p$-mediated regulation of HSC formation and differentiation, we focused on the downstream events of $m i R-142 a-3 p$-irf7 axis. irf7 is a member of the irf family, and irf members often function in host defense and inflammation [27-30]. An inflammation pathway might be involved in HSC and myeloid lineage differentiation in zebrafish [31, 32]. Very recently, Hall et al. [31] reported that infection-induced Gcsfr-NO signaling can enhance the expansion of the hematopoietic stem and progenitor cell compartment in zebrafish. To test whether this signaling was involved in the miR-142a-3p-mediated regulation of HSC development, we examined gcsfr, a very important upstream component in inflammation signaling. The expression of gcsfr in the CHT region at $36 \mathrm{hpf}$ was downregulated in the miR-142a-3p morphants or the irf7-overexpressing embryos, and the decrease of $g c s f r$ expression in the $m i R-142 a-3 p$ morphants was partially rescued by co-injection of irf7 MO (Figure 6A), suggesting that gcsfr acts downstream of irf7. The DAF-FM assay [33] showed that NO production was decreased in the miR-142a-3p morphants but was restored by coinjection of irf7 MO (Figure 6B). NO production is also regulated by blood flow-induced Klf2a-eNOS signaling, as we reported previously [34]. To test whether blood flow-induced Klf2a signaling is involved in the defects in HSC development in the miR-142a-3p morphants, we evaluated the expression of klf $2 a$. klf $2 a$ expression was not altered in the miR-142a-3p morphants compared to control embryos (Figure 6C), suggesting that $k l f 2 a$-mediated blood flow did not contribute to the defects in HSC development in miR-142a-3p morphants. In addition, we also employed the rescue approach by treating $m i R-142 a-$ $3 p$ morphants with the Gcsfr downstream NO agonist, S- nitroso N-acetylpenicillamine (SNAP). WISH showed that the expression of runxl in the AGM was partially restored by treating $m i R-142 a-3 p$ morphants with SNAP (Figure 6D). As expected, an injection of $g c s f$ mRNA can also rescue the expression of runxl in miR-142a-3p morphants (Figure 6D). Taken together, these data suggest that $m i R-142 a-3 p$ regulates HSC formation and differentiation, at least in part, through Gcsfr-NO signaling.

miR-142-3p is involved in definitive hematopoiesis in mouse

Because the sequence of $m i R-142-3 p$ is highly conserved in all vertebrates, including mammals (Supplementary information, Figure S1A), we hypothesized that $m m u-m i R-142-3 p$, the mouse homolog of $m i R-142-3 p$, might also function in the development of the HSCs in the AGM region of mouse embryos. To test this possibility, we first examined its expression in the mouse AGM region. The qPCR results clearly showed that miR-142$3 p$ was highly expressed in E11.5 AGM tissues, but it was not detectable in the neighboring non-AGM tissues (Figure 7A).
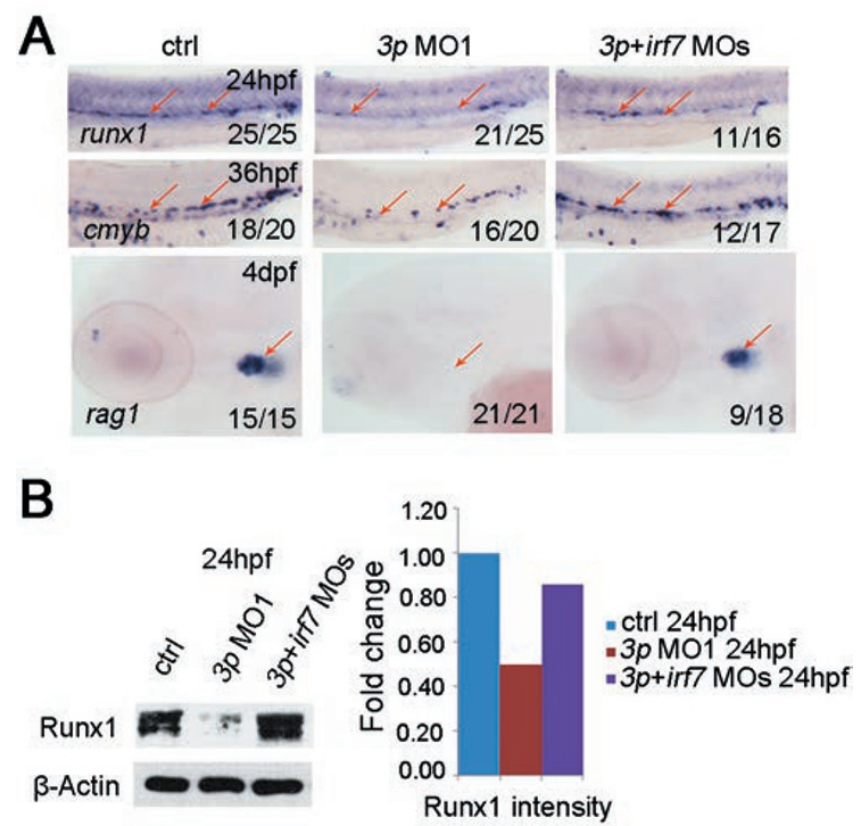

Figure 5 irf7 knockdown can restore the expression of the HSC gene runx1 and T-cell gene rag1 in miR-142a-3p morphants. (A) irf7 knockdown restores the expression of HSC genes runx1 (24 hpf) and cmyb (36 hpf), and the T-cell marker rag1 (4 dpf) in miR-142a-3p morphants, as determined by WISH. (B) irf7 knockdown restores runx 1 expression at the protein level, as shown by western blotting (left panel) and quantitative analysis of the western blotting results (right panel). 
A
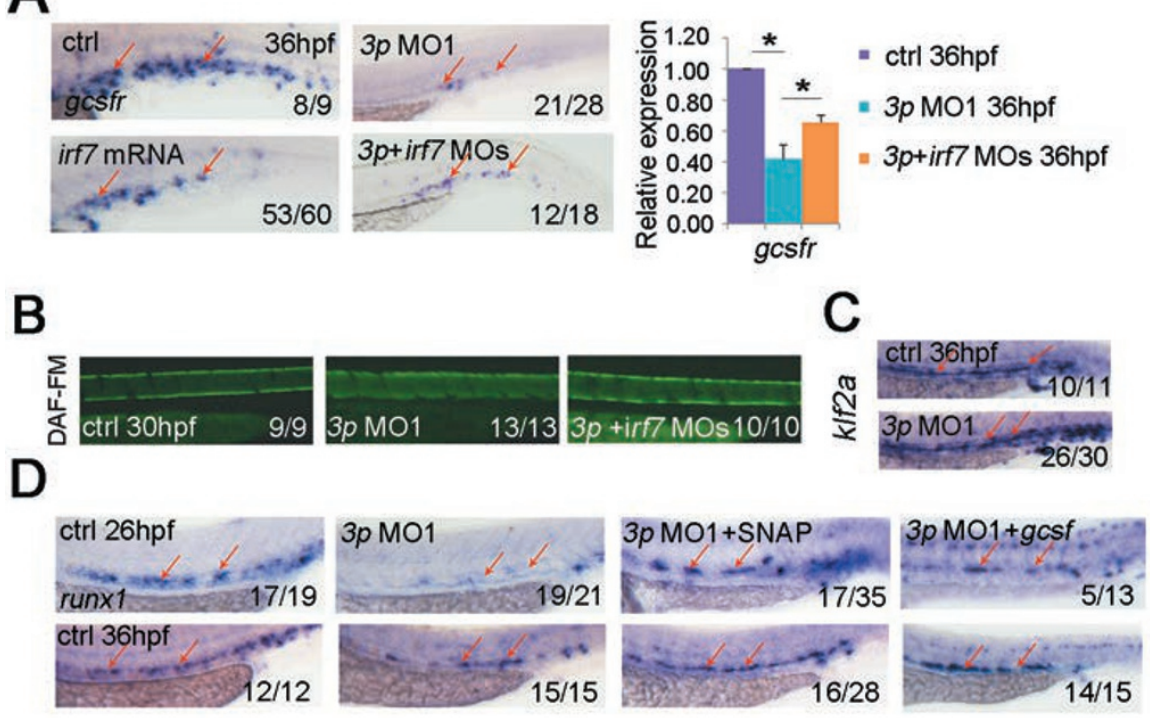

Figure 6 Decreased Gcsfr-NO inflammation signaling might lead to defects in HSC development in miR-142a-3p morphants. (A) Decreased expression of gcsfr in miR-142a-3p morphants and embryos injected with irf7 mRNA at 36 hpf. Co-injection of the irf7 MO partially rescued the gcsfr expression in miR-142a-3p morphants. Left panel, WISH; the red arrows indicate HSCs in the CHT region. Right panel, qPCR showing gcsfr expression in embryos injected with $3 p$ MO1 alone or in combination with irf7 MO (mean $\pm \mathrm{SD}, t$-test, ${ }^{*} P<0.05, n=3$ ). (B) DAF-FM assay performed at 30 hpf showed the decreased production of NO in miR-142a-3p morphants and its restoration by co-injection of irf7 MO. (C) klf2a expression was unaffected in miR-142a-3p morphants at 36 hpf. (D) Partial rescue of runx1 expression was achieved by treating miR-142a-3p morphants with the NO agonist SNAP or by injecting gcsf mRNA. The red arrows indicate runx1 expression in the AGM region.

To determine the functional role of $m i R-142-3 p$ in the HSCs of mouse embryos, we first performed a colonyforming unit-cell (CFU-C) assay in vitro. E11.5 AGMs (41-45 sp) were dissociated using collagenase and the cells were treated with the miR-142-3p duplex (20 pmol) or the $m i R-142-3 p$ inhibitor (40 pmol). Then, the c$\mathrm{Kit}^{+} \mathrm{CD} 34^{+}$cells were sorted and the indicated numbers of single cells were transferred to ultra-low attachment 24-well plates and cultured in CFU-C media. The cells were cultured for 7 days, and based on morphology, the colonies were categorized into BFU-E (burst forming unit-erythroid), CFU-GM (colony forming unit-granulocyte, macrophage), and mixed CFU-GEMM (colony forming unit-granulocyte, erythroid, macrophage, megakaryocyte) or CFU-Mix. We counted the colonies in each category, and found that the addition of the miR-142$3 p$ inhibitor led to a $25 \%$ reduction of the total number of colonies, whereas the $m i R-142-3 p$ duplex caused an approximately 2-fold increase of total colony number (Figure 7B). The proportions of each colony category showed little changes after either treatment (Figure 7B). To confirm the in vitro result, we performed a CFU-S (colony-forming unit-spleen) assay in vivo. As shown in Figure 7C, the number of CFU-S was increased in mice infused with duplex-treated AGM cells, whereas it was decreased in mice infused with inhibitor-treated AGM cells. These data suggest that $m i R-142-3 p$ is important for the expansion of HSCs and their differentiation into lineage-restricted progenitors, such as erythroid and myeloid lineages, both in vitro and in vivo.

To determine whether $m i R-142-3 p$ is also required for the differentiation of the T-lymphoid lineage in mouse, we performed a T-cell differentiation assay in vitro [35]. Dissociated cells from the AGM region were treated with the duplex or inhibitor for $5 \mathrm{~h}$. Then, the sorted c$\mathrm{Kit}^{+} \mathrm{CD} 34^{+}$cells were co-cultured with OP9-DL1 stromal cells and cytokines for 13 days, and the $\mathrm{CD} 25^{+} \mathrm{CD} 44^{+}$ $\mathrm{T}$-cell progenitors were quantified by fluorescenceactivated cell sorting (FACS). The results showed that the duplex increased, whereas the inhibitor decreased, the number of $\mathrm{CD} 25^{+} \mathrm{CD} 44^{+}$cells (Figure 7D), indicating that $m i R-142-3 p$ is required for T-cell differentiation. To investigate whether the expression of $i r f 7$ is also negatively regulated by $m i R-142-3 p$ in mouse, we performed qPCR to quantify the level of irf7 expression after up- or down-regulation of $m i R-142-3 p$ expression. As shown in Figure 7E, the mRNA level of irf7 was decreased after $m i R-142-3 p$ overexpression, whereas it was increased after the inhibition of $m i R-142-3 p$ expression in c$\mathrm{Kit}^{+} \mathrm{CD} 34^{+}$cells, which were dissociated from the E11.5 
AGM and co-cultured with OP9 stromal cells for $48 \mathrm{~h}$. Taken together, these results confirm the hypothesis that $m i R-142-3 p$ is involved in the formation and differentiation of HSCs in the AGM of mouse embryos both in vitro and in vivo, suggesting a highly conserved role of $m i R$ $142-3 p$ in definitive hematopoiesis in vertebrates, from zebrafish to mouse.

\section{Discussion}

Our study reveals that $m i R-142-3 p$ plays pivotal roles in regulating the formation and lineage differentiation of

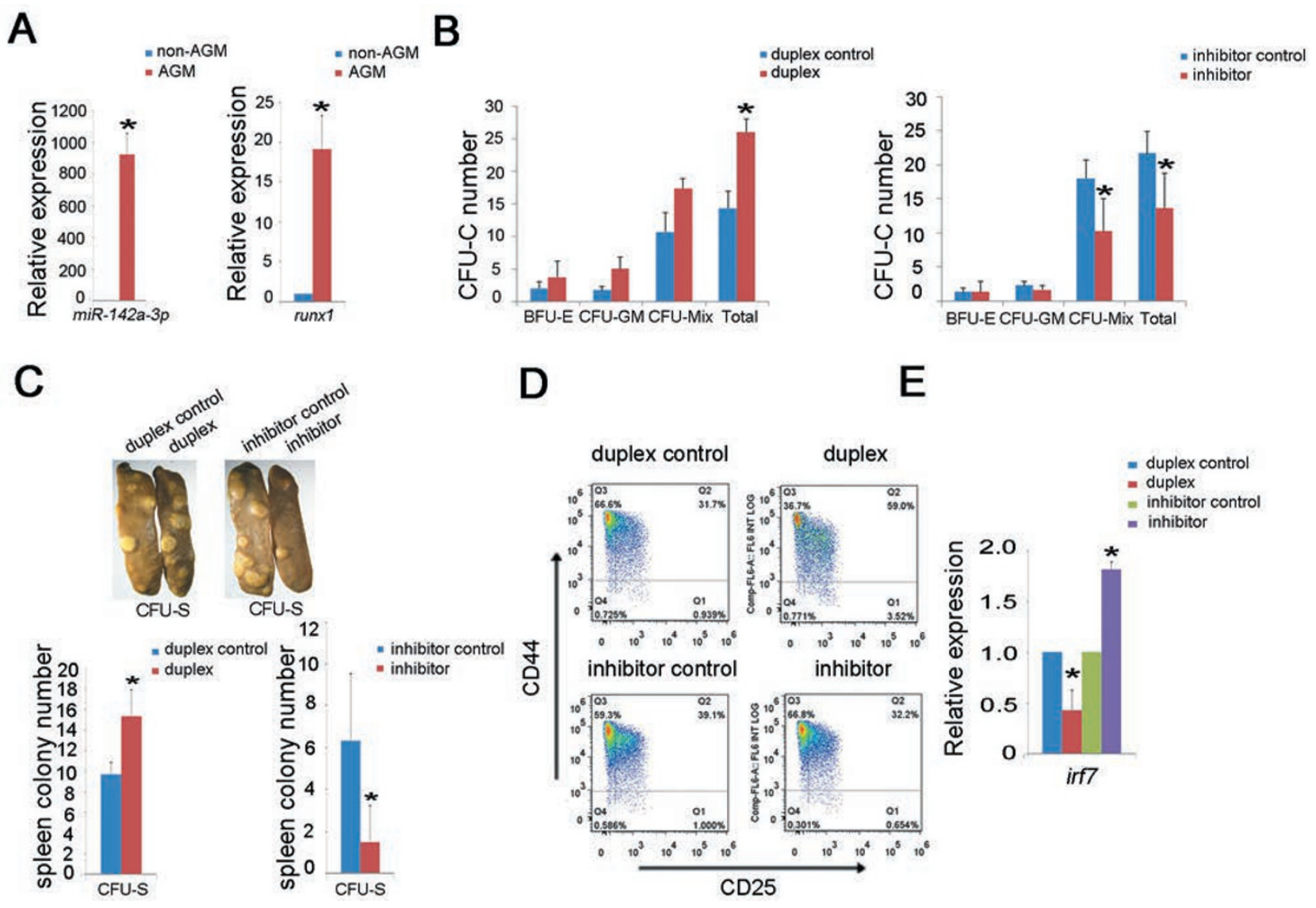

Figure 7 The involvement of $m i R-142-3 p$ in HSC development in mouse. (A) Expression of $m m u-m i R-142-3 p$ in dissected E11.5 AGM tissues and neighboring non-AGM tissues was determined by qPCR (mean $\pm \mathrm{SD}, t$-test, ${ }^{*} P<0.05, n=3$ ). runx 1 was used as a positive control. (B) CFU-C assay. Isolated E11.5 AGM cells were transfected with the miR-142-3p duplex or inhibitor. Five hours after the transfection, the $\mathrm{c}-\mathrm{Kit}^{+} \mathrm{CD} 34^{+}$cells were sorted, and cultured in CFU-C media for 7 days, and several types of colonies formed, including BFU-E, CFU-GM, and CFU-Mix colonies. The mmu-miR-142-3p duplex promoted colony formation, whereas the $m m u-m i R-142-3 p$ inhibitor repressed colony formation. The colonies starting from a seeding density of 800 cells were counted to determine the CFU-C number. Data are presented as mean \pm SD; ${ }^{*} P<0.05$. (C) CFU-S assay. The in vivo splenic colony assay showed that the CFU-S colony number was increased by $\sim 1.5$-fold after the transplantation of duplex-treated cells. However, the number decreased by $\sim 3$-fold after the transplantation of inhibitor-treated cells. Data are presented as mean $\pm \mathrm{SD}$; ${ }^{*} P<0.05$. (D) T-cell differentiation was assessed using FACS. The dissociated cells from the AGM region were treated with the duplex or the inhibitor for $5 \mathrm{~h}$. Then, the sorted c-Kit ${ }^{+} \mathrm{CD} 34^{+}$cells were cultured under T-cell differentiation conditions. The T-lymphoid potential was determined by measuring CD44 and CD25 expression using FACS after 13 days of co-culture with OP9-DL1 cells. The number of lymphoid progenitors increased after duplex treatment while it decreased after inhibitor treatment (duplex control $39 \pm 7 \%$, duplex $53 \pm 6 \%$; inhibitor control $45.5 \pm 7 \%$, inhibitor $38.6 \pm 6 \%)(n=3, P<0.05)$. (E) The expression of irf7 was decreased in c-Kit ${ }^{+} \mathrm{CD} 34^{+}$cells by treatment with the miR-142-3p duplex, while it was increased by treatment with the inhibitor. A representative quantitative RT-PCR result showing the expression of irf7 mRNA in miR-142-3p duplex- and inhibitor-treated c-Kit ${ }^{+} \mathrm{CD} 34^{+}$cells from the E11.5 AGM. Data are presented as mean $\pm \mathrm{SD} ;{ }^{*} P<0.05$. 
the earliest HSCs in vivo during vertebrate embryogenesis. We showed that irf7 acts as a negative regulator of HSC development and differentiation into the T-cell lineages, which can be inhibited by miR-142-3p .

To date, very few miRNAs have been shown to play important roles in HSC development in vertebrates in vivo. miR-142-3p is specifically expressed in HSCs (AGM and $\mathrm{CHT}$ ) and $\mathrm{T}$ cells (thymus) in zebrafish, mouse and other species [15-21]. By knockdown of miR-142a-3p using 2 specific MOs, the numbers of HSCs and T cells were decreased. Interestingly, a recent report using $m i R$ $142^{-1-}$ mice also showed that more $\mathrm{miR}-142^{-1-} \mathrm{CD} 4^{+}$ DCs underwent apoptosis than WT ones [36]. However, defects in HSC development were not examined in that study. Based on our present findings, we speculate that a similar phenotype would be observed at the onset of definitive hematopoiesis in $m i R-142^{-/-} \mathrm{KO}$ mice. Interestingly, a very recent report showed that in Xenopus, $m i R-142-3 p$ is required for HSC lineage specification [37], which is consistent with the results for zebrafish and mouse embryos reported in this study. In contrast, an inverse correlation between $m i R-142-3 p$ expression and HSC development has been suggested recently [20]. Bissels et al. [20] reported that overexpression of miR-142$3 p$ at a dose of $50 \mathrm{nM}$ in human $\mathrm{CD} 133^{+}$cells had a negative effect on their overall colony-forming ability. The apparent difference between those results and ours might be due to the source and age of the cells used (human bone marrow vs mouse AGM; 79 years vs E11.5) and the concentration of $m i R-142-3 p$ utilized for overexpression (50 $\mathrm{nM}$ vs $20 \mathrm{pmol}$ ), which awaits further investigation.

Identifying the targets of $m i R-142-3 p$ is crucial for analyzing the biological function of this miRNA during hematopoiesis. Among the four genes that we investigated, the expression of irf7 was the most upregulated in $m i R-142 a-3 p$ morphants. Reporter assays and $\mathrm{qPCR}$ further indicated that $i r f 7$ is a direct target of $m i R-142 a$ $3 p$. More importantly, the irf7 MO injection rescued the defects in HSC development and T-cell differentiation in the miR-142a-3p morphants. Members of the interferon regulatory factor (IRF) family of transcription factors mainly act as regulators of host defense and inflammation [27-30]. Inflammatory activation of early HSCs has been shown to be evolutionarily conserved [38], and a recent report by Hall et al. [31] demonstrated that, in zebrafish, Gcsfr-NO signaling is required for the expansion of HSCs following infection. Among the irf family members, irf8 was shown to be required for myeloid differentiation into macrophages as opposed to neutrophils [39] and for the regulation of B-cell lineage specification and differentiation [40]. Here, we reveal that knocking down $m i R-142 a-3 p$ expression in zebrafish upregulated the expression of an irf family member, irf7, which might be responsible for the decreased HSC population in the $m i R-142 a-3 p$ morphants. We further showed that irf7 is indeed a direct target of $m i R-142 a-3 p$, which plays a pivotal role in regulating the formation and differentiation of HSCs in zebrafish in vivo and possibly also in mouse, at least in vitro. Further work is required to elucidate how irf7 negatively regulates Gcsfr-NO signaling during HSC development under normal and stressful conditions. However, we cannot exclude the possibility that other target genes of $m i R-142-3 p$ may also be involved in this process.

In summary, our results demonstrate that $m i R-142-3 p$ regulates HSC development by directly targeting $\operatorname{irf} 7$. As $m i R-142-3 p$ and irf7 are highly conserved across species, the newly identified $m i R-142-3 p$-irf7 axis provides new insights into miRNA modulation of HSC formation and differentiation in vertebrates and may provide new clues for the in vitro expansion of therapeutic HSCs.

\section{Materials and Methods}

\section{Fish strains and embryos}

Zebrafish embryos were obtained by the natural spawning of adult $\mathrm{AB}$ strain zebrafish. The embryos were raised and maintained at $28.5^{\circ} \mathrm{C}$ in system water and staged as described previously [41]. The fila:EGFP [42] transgenic line was kindly provided by Steve Wilson (King's College London). The cloche carrier [43], $c m y b$ :EGFP [26], and $k d r l: m C h e r r y / c m y b$ :EGFP [4] lines were kindly provided by Anming Meng (Tsinghua University). This study was approved by the Ethical Review Committee of the Institute of Zoology, Chinese Academy of Sciences, China.

\section{MOs, duplex, mRNA synthesis and microinjection}

Standard MOs and gene-specific antisense MOs were purchased from GeneTools (Philomath, OR) and prepared as $1 \mathrm{mM}$ stock solutions using $\mathrm{ddH}_{2} \mathrm{O}$. The miRNA duplex was purchased from Invitrogen. mRNAs were synthesized using an Ambion mMessage mMachine kit (Ambion, Austin, TX). Standard MOs were used as negative controls in all of the MO injection experiments along with gene-specific MOs. For the fish embryo injections, MOs (2-10 ng), the duplex (5-20 pmol) and capped mRNA were injected separately or in combination into 1- to 2-cell-stage zebrafish embryos at the yolk/blastomere boundary. The MO sequences are listed in Supplementary information, Table S1.

\section{WISH and cryosectioning}

WISH of zebrafish embryos was performed as described previously [34] using probes for myod, pax2.1, ccl25a, klf2a, ikaros, runx 1, cmyb, dltc, flt4, foxn1, rag1, irf7, krt18, foxpla, rxrab and gcsfr. The LNA probe for miR-142a-3p was purchased from EXIQON. The WISH-labeled embryos were treated with $30 \%$ sucrose and then washed three times using PBST and embedded in O.C.T. medium (SAKURA). The embryos were sectioned using LEICA CM1900 cryostats. 


\section{Stem loop RT-PCR, quantitative RT-PCR and western blotting}

The total RNA of embryos from the one-cell stage to $6 \mathrm{dpf}$ was reverse-transcribed using the miR-142a-3p RT-primer 5'-GTCGTATCCAGTGCAGGGTCCGAGGTATTCGCACTGGATACGACTCCATA-3', and the U6 RT primer 5'-AAAAATATGGAGCGCTTCACG-3'. Quantitative RT-PCR and western blotting were performed as described previously [34]. The PCR primers used are listed in Supplementary information, Table S2. All of the experiments were performed in at least triple duplicates in three different biological experiments. The data are given as mean $\pm \mathrm{SD}$. Student's $t$-test was used for statistical comparisons. $P<0.05$ was considered significant and is indicated with one asterisk.

\section{Zebrafish embryo dissociation and FACS}

Embryo dissociation and FACS were performed as described previously by Covassin et al. [44]. The $\mathrm{GFP}^{+}$and $\mathrm{GFP}^{-}$cells were separately collected from $c m y b$ :EGFP and runx1:EGFP transgenic embryos (Zhang P and Liu F, unpublished data) using MoFlo XDP (Beckman). A plasmid containing a runxl intronic enhancer together with a heat-shock minimal promoter fused with EGFP was injected into embryos at the one-cell stage to generate a stable transgenic line.

\section{Chemical treatment}

Embryos at bud stage were treated with SNAP (Sigma-Aldrich) at the concentration of $10 \mu \mathrm{M}$. The treated embryos were examined periodically to ensure that the pharmacologic effect remained constant over time.

\section{Confocal microscopy}

Confocal images were acquired using a Zeiss LSM 510 META confocal laser microscope, and 3D projections were generated using Zeiss LSM software (Carl Zeiss Inc.).

\section{Microarray assay}

The total RNA was extracted from the AGM region of 2- and 4-dpf embryos using TRIzol (Tiangen, Beijing), and Cy3- or Cy5labeled cDNAs from the controls and morphants were hybridized to Agilent Zebrafish Oligo Microarrays (Shanghai Biotechnology Co. Ltd., Shanghai). The expression data were subjected to hierarchical clustering and subsequently depicted in a heat map format.

\section{Reporter assay}

The WT and mutated irf $73^{\prime}$ UTR were cloned and inserted into the EcoRI and SpeI sites of the pGL3-luciferase vector, which was kindly provided by Dr Peifeng Li (Institute of Zoology, CAS, Beijing, China). The mutation sites of irf7 3' UTR were designed as follows: from WT 5'-AGTGG-3' to mut $15^{\prime}$-GACAA-3' and from WT 5'-AACACTATA-3' to mut2 5'-GGTGTCGCC-3'. WT or mutated pGL3-irf7-3' UTR with or without the miR-142a-3p duplex (Invitrogen) were transfected into HEK293T cells using Lipofectamine 2000 (Invitrogen). The luciferase activity was measured using the luciferase activity assay kit (Promega).

\section{DAF-FM assay}

Embryos at $30 \mathrm{hpf}$ were incubated with $5 \mu \mathrm{M}$ DAF-FM diacetate (4-amino-5-methylamino-2', $7^{\prime}$-difluorofluorescein diacetate) for $2 \mathrm{~h}$ at $28.5^{\circ} \mathrm{C}$ and then washed with fish water and observed under a fluorescence microscope.

\section{$C F U-C$ assay and quantitative RT-PCR}

E11.5 mouse AGMs (41-45 sp) were dissociated using collagenase, and the cells were treated with the miR-142-3p duplex (Invitrogen) at $20 \mathrm{pmol}$ and the inhibitor (Invitrogen) at $40 \mathrm{pmol}$. The procedures for siRNA transfection were performed as described in the Lipofectamine 2000 Transfection Reagent protocol (Invitrogen). After $5 \mathrm{~h}$ of transfection, antibody staining was performed for $30 \mathrm{~min}$ at $4{ }^{\circ} \mathrm{C}$ using antibodies specific for CD34 (BD) and c-Kit (eBioscience). The c-Kit ${ }^{+} \mathrm{CD} 34^{+}$cells were sorted using a MoFlo XDP (Beckman Coulter). The indicated numbers of single cells were transferred to ultra-low attachment 24-well plates (COSTAR) and cultured in CFU-C media. The cells were incubated at $37{ }^{\circ} \mathrm{C}$ in $5 \% \mathrm{CO}_{2}$ with $100 \%$ humidity for 7 days, and the number of each type of colony was counted according to their morphology. The experiment was repeated in triplicate. The sequences for the $m i R-142-3 p$ inhibitor and duplex are listed in Supplementary information, Table S2.

After transfection with the miR-142-3p duplex or inhibitor, the FACS-sorted c-Kit ${ }^{+} \mathrm{CD} 34^{+}$cells were cultured on mouse OP9 stromal cells in medium supplemented with hematopoietic cytokines

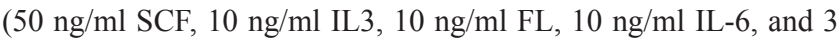
$\mathrm{U} / \mathrm{ml}$ Epo). After being cultured for $48 \mathrm{~h}$, the cells were harvested for quantitative PCR to evaluate the expression of $m i R-142-3 p$ and irf7.

\section{CFU-S assay}

The AGM regions of E11.5 mouse embryos were dissected, and the cells were suspended as single cells in solution, and then were treated with the miR-142-3p duplex (20 pmol) or inhibitor (40 pmol). After $5 \mathrm{~h}$ transfection, one embryo equivalent was transplanted via the tail vein. There were four individual mice in each experimental group. The spleens were obtained 8 days posttransplantation, and the number of colonies formed was counted under a stereomicroscope.

\section{T lymphoid progenitor differentiation assay}

An equal number of c-Kit ${ }^{+} \mathrm{CD} 34^{+}$cells and OP9-DL1 stromal cells were co-cultured in $\alpha$-MEM supplemented with $15 \% \mathrm{FBS}$, $50 \mathrm{ng} / \mathrm{ml} \mathrm{SCF}, 10 \mathrm{ng} / \mathrm{ml} \mathrm{IL7}$, and $20 \mathrm{ng} / \mathrm{ml} \mathrm{FL-3.} \mathrm{After} 13$ days of co-culture, the cells were labeled with CD25-PE and CD44-APC antibodies and analyzed using flow cytometry.

\section{Statistical analysis}

For statistical analysis, Student's unpaired two-tailed $t$-test was used for all of the comparisons unless otherwise indicated.

\section{Acknowledgments}

We thank the laboratory members for helpful discussions and critical reading of the paper. This work was supported by the National Basic Research Program of China (2010CB945300, 2011CB943900, and 2012CB945101), the National Natural Science Foundation of China (31271570, 90919055), and the Strategic Priority Research Program of the Chinese Academy of Sciences (XDA01010110). 


\section{References}

1 Zhang C, Patient R, Liu F. Hematopoietic stem cell development and regulatory signaling in zebrafish. Biochim Biophys Acta 2013; 1830:2370-2374.

2 Orkin SH, Zon LI. Hematopoiesis: an evolving paradigm for stem cell biology. Cell 2008; 132:631-644.

3 Kissa K, Herbomel P. Blood stem cells emerge from aortic endothelium by a novel type of cell transition. Nature 2010; 464:112-115.

4 Bertrand JY, Chi NC, Santoso B, et al. Haematopoietic stem cells derive directly from aortic endothelium during development. Nature 2010; 464:108-111.

5 Bartel DP. MicroRNAs: genomics, biogenesis, mechanism, and function. Cell 2004; 116:281-297.

6 Forman JJ, Coller HA. The code within the code: microRNAs target coding regions. Cell Cycle 2010; 9:1533-1541.

7 O'Connell RM, Baltimore D. MicroRNAs and hematopoietic cell development. Curr Top Dev Biol 2012; 99:145-174.

8 Bissels U, Bosio A, Wagner W. MicroRNAs are shaping the hematopoietic landscape. Haematologica 2012; 97:160-167.

9 Lechman ER, Gentner B, van Galen P, et al. Attenuation of miR-126 activity expands HSC in vivo without exhaustion. Cell Stem Cell 2012; 11:799-811.

10 Grabher C, Payne EM, Johnston AB, et al. Zebrafish microRNA-126 determines hematopoietic cell fate through c-Myb. Leukemia 2011; 25:506-514.

11 Dore LC, Amigo JD, Dos Santos CO, et al. A GATA-1-regulated microRNA locus essential for erythropoiesis. Proc Natl Acad Sci USA 2008; 105:3333-3338.

$12 \mathrm{Fu}$ YF, Du TT, Dong M, et al. Mir-144 selectively regulates embryonic alpha-hemoglobin synthesis during primitive erythropoiesis. Blood 2009; 113:1340-1349.

13 Pase L, Layton JE, Kloosterman WP, et al. miR-451 regulates zebrafish erythroid maturation in vivo via its target gata2. Blood 2009; 113:1794-1804.

14 Ramkissoon SH, Mainwaring LA, Ogasawara Y, et al. Hematopoietic-specific microRNA expression in human cells. Leuk Res 2006; 30:643-647.

15 Chen CZ, Li L, Lodish HF, Bartel DP. MicroRNAs modulate hematopoietic lineage differentiation. Science 2004; 303:8386.

16 Reddy AM, Zheng Y, Jagadeeswaran G, et al. Cloning, characterization and expression analysis of porcine microRNAs. BMC Genomics 2009; 10:65.

17 Liao R, Sun J, Zhang L, et al. MicroRNAs play a role in the development of human hematopoietic stem cells. J Cell Biochem 2008; 104:805-817.

18 Jin P, Wang E, Ren J, et al. Differentiation of two types of mobilized peripheral blood stem cells by microRNA and cDNA expression analysis. $J$ Transl Med 2008; 6:39.

19 Sun W, Shen W, Yang S, et al. miR-223 and miR-142 attenuate hematopoietic cell proliferation, and miR-223 positively regulates miR-142 through LMO2 isoforms and CEBP-beta. Cell Res 2010; 20:1158-1169.

20 Bissels U, Wild S, Tomiuk S, et al. Combined characterization of microRNA and mRNA profiles delineates early differentiation pathways of $\mathrm{CD} 133^{+}$and $\mathrm{CD} 34^{+}$hematopoietic stem and progenitor cells. Stem Cells 2011; 29:847-857.
21 Yuan W, Sun W, Yang S, et al. Downregulation of microRNA-142 by proto-oncogene LMO2 and its co-factors. Leukemia 2008; 22:1067-1071.

22 Wang XS, Gong JN, Yu J, et al. MicroRNA-29a and microRNA-142-3p are regulators of myeloid differentiation and acute myeloid leukemia. Blood 2012; 119:4992-5004.

23 Sun Y, Varambally S, Maher CA, et al. Targeting of microRNA-142-3p in dendritic cells regulates endotoxin-induced mortality. Blood 2011; 117:6172-6183.

24 Nishiyama T, Kaneda R, Ono T, et al. miR-142-3p is essential for hematopoiesis and affects cardiac cell fate in zebrafish. Biochem Biophys Res Commun 2012; 425:755-761.

25 Lalwani MK, Sharma M, Singh AR, et al. Reverse genetics screen in zebrafish identifies a role of miR-142a-3p in vascular development and integrity. PLoS One 2012; 7:e52588.

26 North TE, Goessling W, Walkley CR, et al. Prostaglandin E2 regulates vertebrate haematopoietic stem cell homeostasis. $\mathrm{Na}$ ture 2007; 447:1007-1011.

27 Ozato K, Tailor P, Kubota T. The interferon regulatory factor family in host defense: mechanism of action. $J$ Biol Chem 2007; 282:20065-20069.

28 Balaraman S, Tewary P, Singh VK, Madhubala R. Leishmania donovani induces interferon regulatory factor in murine macrophages: a host defense response. Biochem Biophys Res Commun 2004; 317:639-647.

29 Fredericksen BL, Gale M Jr. West Nile virus evades activation of interferon regulatory factor 3 through RIG-I-dependent and -independent pathways without antagonizing host defense signaling. J Virol 2006; 80:2913-2923.

30 Tamura T, Ozato K. ICSBP/IRF-8: its regulatory roles in the development of myeloid cells. J Interferon Cytokine Res 2002; 22:145-152.

31 Hall CJ, Flores MV, Oehlers SH, et al. Infection-responsive expansion of the hematopoietic stem and progenitor cell compartment in zebrafish is dependent upon inducible nitric oxide. Cell Stem Cell 2012; 10:198-209.

32 Liongue C, Hall CJ, O'Connell BA, Crosier P, Ward AC. Zebrafish granulocyte colony-stimulating factor receptor signaling promotes myelopoiesis and myeloid cell migration. Blood 2009; 113:2535-2546.

33 North TE, Goessling W, Peeters M, et al. Hematopoietic stem cell development is dependent on blood flow. Cell 2009; 137:736-748

34 Wang L, Zhang P, Wei Y, et al. A blood flow-dependent klf2aNO signaling cascade is required for stabilization of hematopoietic stem cell programming in zebrafish embryos. Blood 2011; 118:4102-4110

35 Li Z, Lan Y, He W, et al. Mouse embryonic head as a site for hematopoietic stem cell development. Cell Stem Cell 2012; 11:663-675.

36 Mildner A, Chapnik E, Manor O, et al. Mononuclear phagocyte miRNome analysis identifies miR-142 as critical regulator of murine dendritic cell homeostasis. Blood 2013; 121:10161027.

37 Nimmo R, Ciau-Uitz A, Ruiz-Herguido C, et al. miR-142-3p controls the specification of definitive hemangioblasts during ontogeny. Dev Cell 2013; 26:237-249.

38 Takizawa H, Boettcher S, Manz MG. Demand-adapted regulation of early hematopoiesis in infection and inflammation. 
Blood 2012; 119:2991-3002.

$39 \mathrm{Li} \mathrm{L}$, Jin $\mathrm{H}, \mathrm{Xu}$ J, et al. Irf8 regulates macrophage versus neutrophil fate during zebrafish primitive myelopoiesis. Blood 2011; 117:1359-1369.

40 Wang $\mathrm{H}$, Lee $\mathrm{CH}$, Qi C, et al. IRF8 regulates B-cell lineage specification, commitment, and differentiation. Blood 2008; 112:4028-4038.

41 Kimmel CB, Ballard WW, Kimmel SR, Ullmann B, Schilling TF. Stages of embryonic development of the zebrafish. Dev Dyn 1995; 203:253-310.

42 Lawson ND, Weinstein BM. In vivo imaging of embryonic vascular development using transgenic zebrafish. Dev Biol 2002; 248:307-318.

43 Stainier DY, Weinstein BM, Detrich HW III, et al. Cloche, an early acting zebrafish gene, is required by both the endothelial and hematopoietic lineages. Development 1995; 121:31413150 .

44 Covassin L, Amigo JD, Suzuki K, et al. Global analysis of hematopoietic and vascular endothelial gene expression by tissue specific microarray profiling in zebrafish. Dev Biol 2006; 299:551-562.

(Supplementary information is linked to the online version of the paper on the Cell Research website.) 